\title{
Theory for nonlinear dynamic force spectroscopy
}

\author{
Oscar Björnham ${ }^{1}$ Magnus Andersson ${ }^{2,3}$ (i)
}

Received: 19 May 2016 / Revised: 1 July 2016 / Accepted: 11 July 2016 / Published online: 26 July 2016

(C) The Author(s) 2016. This article is published with open access at Springerlink.com

\begin{abstract}
Dynamic force spectroscopy (DFS) is an experimental technique that is commonly used to assess information on the strength, energy landscape, and lifetime of noncovalent bio-molecular interactions. DFS traditionally requires an applied force that increases linearly with time so that the bio-complex under investigation is exposed to a constant loading rate. However, tethers or polymers can modulate the applied force in a nonlinear manner. For example, bacterial adhesion pili and polymers with wormlike chain properties are structures that show nonlinear force responses. In these situations, the theory for traditional DFS cannot be readily applied. In this work, we expand the theory for DFS to also include nonlinear external forces while still maintaining compatibility with the linear DFS theory. To validate the theory, we modeled a biocomplex expressed on a stiff, an elastic, and a worm-like chain polymer, using Monte Carlo methods, and assessed the corresponding rupture force spectra. It was found that the nonlinear DFS (NLDFS) theory correctly predicted the numerical results. We also present a protocol suggesting an experimental approach and analysis method of the data to estimate the bond length and the thermal off-rate.
\end{abstract}

Keywords AFM $\cdot$ Optical tweezers $\cdot$ Receptor $\cdot$ Ligand

Magnus Andersson

magnus.andersson@umu.se

1 Swedish Defence Research Agency (FOI), 90621 Umeå, Sweden

2 Department of Physics, Umeå University, 90187 Umeå, Sweden

3 Umeå Center for Molecular Research, Umeå University, 90187 Umeå, Sweden

\section{Introduction}

In the late 1990s, Evans et al. formulated the theoretical basis for dynamic force spectroscopy (DFS) (Evans and Ritchie 1997; Merkel et al. 1999) that could allow for a time-varying force in the expression for bond dissociation originally given by Kramers (1940), and later refined by Bell (1978). Since then, DFS has successfully been employed in a wide range of applications in the field of biophysics to assess information on the strength, energy landscape, and lifetime of bio-molecular interactions (receptor-ligand bonds) at the molecular scale. DFS is commonly performed using force transducers such as optical tweezers (OT) or atomic force microscopy (AFM) instruments, which can apply loading rates from a few $\mathrm{pN} / \mathrm{s}$ to several $\mathrm{nN} / \mathrm{s}$. Using these techniques and the theory for DFS, atomic information on, for example, the Streptavidin-Biotin complex (Lee et al. 1994; Yuan et al. 2000), the digoxigenin-antibody complex (Neuert et al. 2006), the Mucin 1-antibody bond (Sulchek et al. 2005), and the unbinding force of complementary DNA (Strunz et al. 1999) have been revealed.

Although the theory is very useful in its current state, a restriction of the DFS theory is the requirement of a force that increases linearly with time. An experimental system can often be configured to provide an external force that meets this constraint for a limited force interval. However, for some complexes under study, there can be situations when a more general time dependency of the external force in the DFS theory is required. For example, when the rupture force surpasses the linear span of the probe in a force spectroscopy apparatus or when measurements on bonds connected to biological tissues or organelles that have an intrinsic nonlinear response to external forces are investigated. 
Many bacterial adhesion organelles, commonly called pili, expressed by uropathogenic, enterotoxigenic, and respiratory tract associated bacteria exhibit nonlinear force responses (Forero et al. 2006; Miller et al. 2006; Andersson et al. 2007; Chen et al. 2011; Castelain et al. 2011; Mortezaei et al. 2015a, b). In particular, extensions of pili expressed by uropathogenic and enterotoxigenic bacteria show distinct nonlinear force responses, i.e., they respond to an external force initially by unwinding the structure at a constant force and thereafter by a pseudo-elastic force response. A nonlinear force response, similar to that of a worm-like chain (WLC) polymer, is also seen when extending T4 adhesion pili expressed by Streptococcus pneumoniae (Castelain et al. 2009). The response from these adhesion organelles are thus nonlinear with extension by nature. To assess information on the adhesin using DFS, without using a force feedback system, the theory needs to be refined.

In this work, we extend the DFS theory to include nonlinear external forces. The theory, referred to as nonlinear dynamic force spectroscopy (NLDFS), covers positive loading rates in a nonlinear regime where the force increases continuously. NLDFS is compatible with the linear DFS theory, which is shown to be a special case. We validated NLDFS by modeling an adhesin expressed on polymers that exhibit different nonlinear force responses using Monte Carlo simulations, and by assessing the rupture force spectra for various extension velocities. The results, for all tested cases, show that the nonlinear DFS theory correctly predicts the most probable rupture force obtained from the stochastic modeled data and that the methodology suggested in this work is applicable to a variety of experimental investigations.

\section{Theory}

\section{Linear dynamic force spectroscopy}

We start by briefly introducing the traditional DFS theory. For detailed information, see (Evans 2001). Kramers (1940) used the Smoluchowski equation to conclude that the transition rate, i.e., the thermal off-rate $k_{\text {off }}^{\text {th }}$, which gives information on how frequently a bond transits between two states separated by an energy barrier $E_{T}$, can be described by an Arrhenius factor,

$k_{\mathrm{off}}^{\mathrm{th}}=v_{\mathrm{a}} e^{-\frac{E_{T}}{k_{\mathrm{B}} T}}$,

where $v_{\mathrm{a}}$ is the attempt rate originating from the molecular vibrations in an overdamped condensed system, $k_{\mathrm{B}}$ is the Boltzmann's constant, and $T$ is the absolute temperature. Almost 40 years later, Bell (1978) inserted the effect of an external force, $F$, to the theory of Kramers to obtain an expression for the force-dependent dissociation rate $k_{\text {off }}$, given by $k_{\mathrm{off}}=k_{\mathrm{off}}^{\mathrm{th}} e^{\frac{F x_{\mathrm{b}}}{k_{\mathrm{B}} T}}$,

where $x_{\mathrm{b}}$ is the bond length that describes the spatial distance between the energy minima and the transition energy barrier. Evans et al. extended Bell's work by including a linearly increasing force described by a constant loading rate defined as the time derivative of the force, $r(1-3)$. They showed that with increasing force, the probability for the bond to rupture increases. This implies that there will be a maximum likelihood for bond rupture for a specific force. This force, which often is referred to as the most probable rupture force, $F^{*}$, depends on the bond length, the thermal off-rate, and the loading rate and is found as the peak force in a rupture force spectrum. We will refer to this entity as the peak force throughout the rest of this work. Evans et al. showed that the peak force can be explicitly expressed as

$F^{*}=\frac{k_{\mathrm{B}} T}{x_{\mathrm{b}}} \ln \left(\frac{r x_{\mathrm{b}}}{k_{\mathrm{off}}^{\mathrm{th}} k_{\mathrm{B}} T}\right)$.

\section{Nonlinear dynamic force spectroscopy}

To reduce the complexity when analyzing experimental data obtained from nonlinear loading rates, we restrict the range of the nonlinear theory to continuous forces with positive time-dependent loading rates. This implies that the force is always increasing and that every force value is only present once. This restriction does not effectively limit the usability of NLDFS since in a force spectroscopy experiment the system under study is not likely to be exposed to external forces with alternating negative and positive loading rates.

To derive the necessary equations for NLDFS, we start by considering the probability $P$ of an intact bond. The probability rate of bond rupture equals the negative change of $P$ over time and can be expressed as

$-\frac{\mathrm{d} P}{\mathrm{~d} t}=-\frac{\mathrm{d} P}{\mathrm{~d} F} r=k_{\mathrm{off}} P$,

where $r$ is the loading rate defined as

$r \equiv \frac{\mathrm{d} F}{\mathrm{~d} t}$.

The derivative of the probability rate, which is indicative of the position of the peak force, can thereby be expressed in two ways, viz. as

$\frac{\mathrm{d}^{2} P}{\mathrm{~d} t^{2}}=-\frac{\mathrm{d}\left(k_{\mathrm{off}} P\right)}{\mathrm{d} t}=-\frac{\mathrm{d} k_{\mathrm{off}}}{\mathrm{d} t} P-k_{\mathrm{off}} \frac{\mathrm{d} P}{\mathrm{~d} t}=P\left(k_{\mathrm{off}}^{2}-\frac{\mathrm{d} k_{\mathrm{off}}}{\mathrm{d} t}\right)$,

and as 


$$
\begin{aligned}
\frac{\mathrm{d}^{2} P}{\mathrm{~d} t^{2}} & =\frac{\mathrm{d}}{\mathrm{d} t}\left(\frac{\mathrm{d} P}{\mathrm{~d} F} r\right)=\frac{\mathrm{d}}{\mathrm{d} t}\left(\frac{\mathrm{d} P}{\mathrm{~d} F}\right) r+\frac{\mathrm{d} P}{\mathrm{~d} F} \frac{\mathrm{d} r}{\mathrm{~d} t} \\
& =\frac{\mathrm{d}}{\mathrm{d} F}\left(\frac{\mathrm{d} P}{\mathrm{~d} F}\right) r^{2}+\frac{\mathrm{d} P}{\mathrm{~d} F} \frac{\mathrm{d} r}{\mathrm{~d} t} .
\end{aligned}
$$

The term

$$
\frac{\mathrm{d}}{\mathrm{d} F}\left(\frac{\mathrm{d} P}{\mathrm{~d} F}\right) r^{2}
$$

is zero at the peak force, which implies that it is possible to combine Eqs. (6) and (7) to obtain the following relationship at the peak of the force rupture spectrum,

$\frac{\mathrm{d} P}{\mathrm{~d} F} \frac{\mathrm{d} r}{\mathrm{~d} t}=P\left(k_{\mathrm{off}}^{2}-\frac{\mathrm{d} k_{\mathrm{off}}}{\mathrm{d} t}\right)$.

Thus by inserting the expression for $\mathrm{d} P / \mathrm{d} F$ from Eq. (4) into Eq. (8), we obtain

$\frac{1}{r} \frac{\mathrm{d} r}{\mathrm{~d} t}=\frac{1}{k_{\mathrm{off}}} \frac{\mathrm{d} k_{\mathrm{off}}}{\mathrm{d} t}-k_{\mathrm{off}}$,

which, in turn, can be written as

$\frac{\mathrm{d} \ln r}{\mathrm{~d} t}=\frac{\mathrm{d} \ln k_{\text {off }}}{\mathrm{d} t}-k_{\text {off }}$.

Making use of the expression for the dissociation rate, Eq. (2), gives the resulting relation between the loading rate and the peak force as

$\frac{\mathrm{d} \ln r}{\mathrm{~d} t}=\frac{r x_{\mathrm{b}}}{k_{\mathrm{B}} T}-k_{\mathrm{off}}^{\text {th }} e^{\frac{F^{*} x_{\mathrm{b}}}{k_{\mathrm{B}} T}}$,

which can be reformulated as

$F^{*}=\frac{k_{\mathrm{B}} T}{x_{\mathrm{b}}} \ln \left[\frac{1}{k_{\mathrm{off}}^{\mathrm{th}}}\left(\frac{r x_{\mathrm{b}}}{k_{\mathrm{B}} T}-\frac{\mathrm{d} \ln r}{\mathrm{~d} t}\right)\right]$.

\section{Wormlike chain model}

We used the WLC model as a case study to evaluate the NLDFS theory. The WLC model is commonly used to describe the nonlinear entropic driven force response of biopolymers exposed to external forces (Strick et al. 2002; Kiss et al. 2006; Bianco et al. 2007; Björnham et al. 2008; Björnham and Schedin 2009). In this model, the force can be expressed as a function of the distance between the two ends of the polymer, which, for the inelastic case, is given by

$F=\frac{k_{\mathrm{B}} T}{l_{\mathrm{p}}}\left[\frac{1}{4}\left(1-\frac{L}{L_{\mathrm{c}}}\right)^{-2}-\frac{1}{4}+\frac{L}{L_{\mathrm{c}}}\right]$,

where $l_{\mathrm{p}}$ is the persistence length, $L$ is the Euclidian distance between the two ends of the molecule, and $L_{\mathrm{c}}$ is the contour length of the polymer. The contour length is the structural length of the polymer and equals $L$ if the polymer is fully stretched. If the polymer is extended at a constant velocity, $v$, the parameter $L$ can be expressed as

$L=v t$.

This implies that the effective loading rate can be given as

$r(t) \equiv \frac{\mathrm{d} F(t)}{\mathrm{d} t}=\frac{\mathrm{d} F}{\mathrm{~d} L} \frac{\mathrm{d} L}{\mathrm{~d} t}=\frac{k_{\mathrm{B}} T}{l_{\mathrm{p}}} \frac{v}{L_{\mathrm{c}}}\left[\frac{1}{2}\left(1-\frac{v t}{L_{\mathrm{c}}}\right)^{-3}+1\right]$.

The NLDFS theory then predicts that the peak force should be given by Eq. (12) with $r(t)$ being given by Eq. (15) for WLC.

\section{Results and discussion}

\section{Validation of the NLDFS theory}

To investigate the validity of the NLDFS theory for different time-dependent external forces, it was compared to the analytic solution for the rupture probability, i.e., Eq. (4) together with Eq. (2). Further on, numerical simulations by means of Monte Carlo (MC) methods were conducted and used as validation. We set the bond length, $x_{\mathrm{b}}$, to $0.70 \mathrm{~nm}$ and the thermal off-rate, $k_{\text {off }}^{\text {th }}$, to $10^{-4} / \mathrm{s}$, which are values in the typical range for noncovalent adhesion bonds (Sulchek et al. 2005; Björnham et al. 2009). The bio-complex was exposed to three different force responses all with an extension velocity of $10.0 \mu \mathrm{m} / \mathrm{s}$. To verify the simulation and the analysis procedure, three sets of data with one million measurements each were compiled using a narrow Gaussian kernel with a width of $0.50 \mathrm{pN}$ (see Fig. 1). The Gaussian kernel function will push the peak of the distribution slightly towards lower forces, since the analytic distribution is skewed. Although the effect is negligible here, it is recommended, in both DFS and NLDFS, to carefully choose the width of the Gaussian kernel to minimize this effect and at the same time obtain a smooth curve to identify the peak force.

In the first case, the force was increased linearly with time, thus resulting in a constant loading rate. This implies that Eq. (12) is reduced to Eq. (3), which is the commonly used expression for the peak force in linear DFS. For the case with a loading rate of $100 \mathrm{pN} / \mathrm{s}$, Fig. 1a shows the resulting rupture force probability spectrum. The inset shows, qualitatively, the time evolution of the force. As can be seen, the analytical solution (black dashed line), which according to Eq. (12) is $70.7 \mathrm{pN}$, coincides perfectly with that of the Monte Carlo simulations (red line). Moreover, the predicted peak force (green vertical dashed line), using 

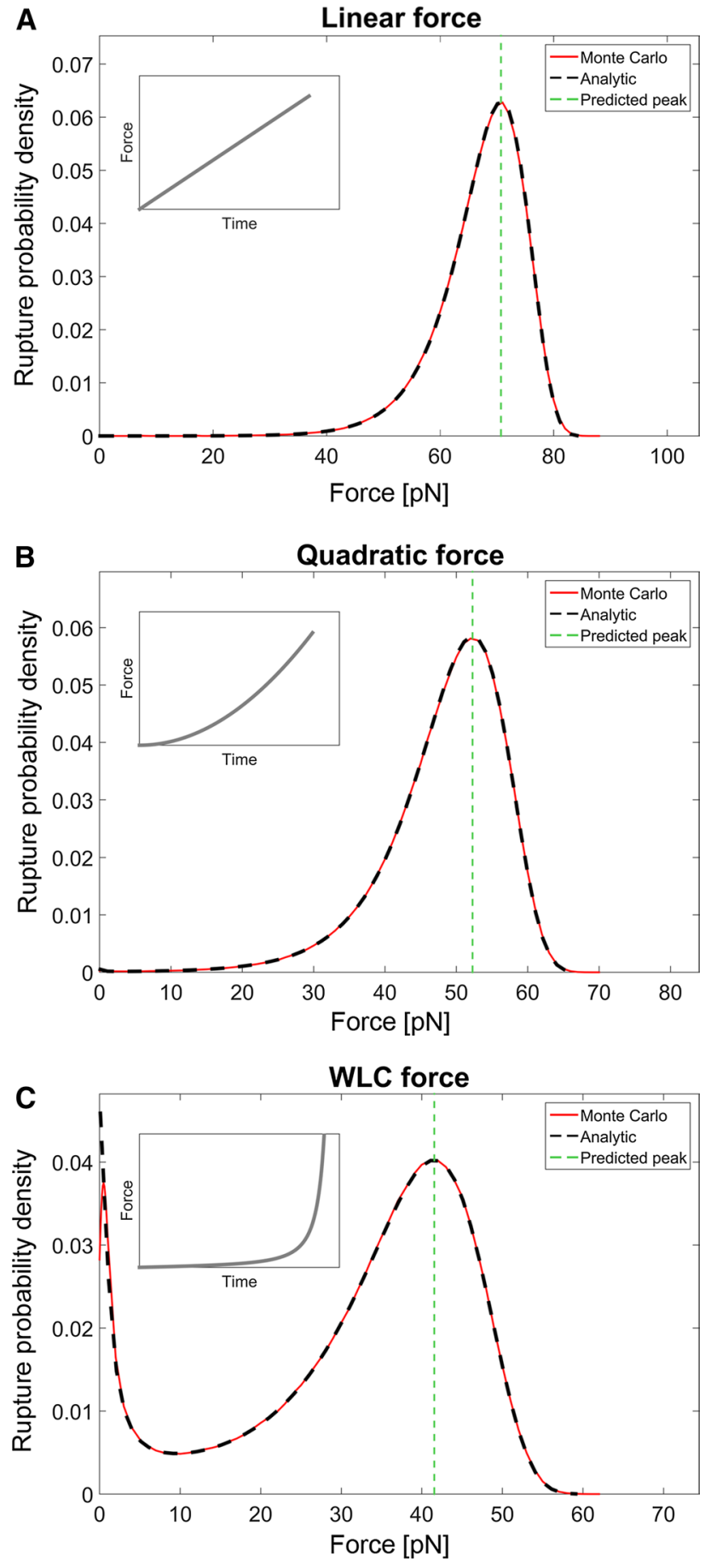

Eq. (12), matches the force for which the distribution has a maximum of both curves.

To model nonlinear increasing forces, e.g., to mimic cases when a receptor-ligand pair is attached to a membrane or polymer, we applied both a quadratically increasing force (elastic reversible polymer) and a force that follows that of a WLC model, i.e., Equation (13). Figure 1b, c, respectively, display the rupture probability densities from the simulations using these two nonlinear forces. The two
Fig. 1 An example of the rupture probability distribution using one million samples for a velocity of $10.0 \mu \mathrm{m} / \mathrm{s}$. The black dashed line is the analytical solution while the red line is the density estimate from the Monte Carlo simulations, using a Gaussian kernel with standard deviation of $0.50 \mathrm{pN}$. The vertical green line is the peak force, $F^{*}$, predicted by Eq. (12). The agreement is excellent except for a small deviation at the smallest forces in the WLC-case due to inherent properties of the kernel density estimation method at the boundary of the interval. The inset figure depicts the relation of the applied force with respect to time. For the linear and quadratic cases, the force was given by $F=a_{1} x$ and $F=a_{2} x^{2}$ with the constants $a_{1}$ and $a_{2}$ set to $10 \mathrm{pN} / \mu \mathrm{m}$ and $10^{-3} \mathrm{pN} / \mu \mathrm{m}^{2}$, respectively. For the WLC case the force was given by Eqs. (13) and (14) with $L_{\mathrm{c}}=10.0 \mu \mathrm{m}$ and $l_{\mathrm{p}}=3.00 \mathrm{~nm}$

panels show that the peak forces predicted by the theory agree with that of the simulations for both the case with a quadratically increasing force, $52.3 \mathrm{pN}$, and for the case with the WLC, $41.6 \mathrm{pN}$. Since the peak forces predicted by the theory given above are in good agreement with those of the numerical solutions for all force curves, we conclude that the NLDFS theory can accurately predict the peak force of a receptor-ligand pair connected with polymers showing linear as well as nonlinear force responses.

Note that in Fig. 1c, when bonds are linked via WLC polymers, the rupture probability curve shows two peaks. These two peaks can be explained by the initial slowly increasing force and the final rapidly increasing force experienced by bonds linked to WLC polymers that are extended. Thus, two effective loading rates are present. A small fraction of bonds break at the lower regime and the rest break at the higher regime.

\section{Applying NLDFS theory to experimental data}

In practice, however, it is of limited use to calculate the peak force solely for the cases when the bond length and the thermal off-rate are known a priori. Instead, the theory must be able to serve as a tool for experimentalists to estimate these two parameters from measurement data. The question is then: how do you design an experiment protocol to extract the desired parameter values?

\section{DFS}

DFS provides a technique to assess the bond length and the thermal off-rate following a straightforward scheme. First, the bio-complex under study is exposed to an external force that increases linearly with time, i.e., with a constant loading rate. Eventually the bond will break, giving rise to a rupture force. However, a single rupture force is solely one sample from the probability distribution that represents the specific loading rate, the bond length, and the thermal off-rate. To obtain sufficient statistics to quantify the distribution, the rupture force must be sampled many times 
for a given loading rate. Second, the rupture force spectrum is constructed from the set of rupture forces obtained, whereby the peak force is identified by localizing the peak of the distribution. Third, the loading rate is changed and a new set of measurements are conducted resulting in a new value of $F^{*}$. Thus, for every loading rate, a corresponding value of the peak force is given. The bond length and the thermal off-rate can thereafter be found by fitting Eq. (3) to this set of data. This is a well-working method that has been widely used. However, as was alluded to above, a limitation of DFS is the constraint that Eq. (3) is valid only under the assumption of constant loading rates.

\section{NLDFS}

As described above, DFS utilizes constant values of the loading rates in the experiments. These values of loading rates are used in combination with their corresponding values of the peak forces to find estimates of the bond length and the thermal off-rate.

In NLDFS, the loading rate is not constant during a measurement, which therefore requires a slightly different approach. Instead of keeping the loading rate constant, the pulling velocity, $v$, of the force transducer is held constant during a measurement. Hence, the rupture forces are recorded as for DFS but the peak force is paired with the corresponding velocity. The velocity is then changed and a new set of measurements is performed. Thus, for each velocity there is a corresponding value of the peak force. These data are used together with Eq. (12) to obtain the estimated parameter values. However, to do this, an expression for the loading rate as a function of the velocity needs to be derived. For the case when the force only depends on the position, i.e., $F=F(L)$, this can be done in the following way. First, it should be noted that the loading rate can be written as

$r \equiv \frac{\mathrm{d} F}{\mathrm{~d} t}=\frac{\mathrm{d} F}{\mathrm{~d} L} \frac{\mathrm{d} L}{\mathrm{~d} t}=\frac{\mathrm{d} F}{\mathrm{~d} L} v$

where $L$ now is a measure of the position of the force transducer, given by $v t$ where both $v$ and $t$ are known entities. The derivative $\mathrm{d} F / \mathrm{d} L$ is in general a function of $L$ that needs to be known or assessed, which can be found either through theoretic consideration of the system or by using measurement data. When this relation is established, Eq. (12) provides a full prediction of the expected value of the peak force given the velocity, the bond length and the thermal off-rate. Even though Eq. (12) might turn out to be an implicit function, the solution for the peak force can readily be found. This means that for every combination of the parameter values there will be one theoretical and one measured value of the peak force. Standard algorithms may then by utilized to find parameter values that minimize the mean square error of these forces for all velocities.

\section{Protocol for NLDFS}

A general description of how to obtain the bond length and the thermal off-rate using measurements was shown above. We will here give a more explicit protocol how this could be done in practice. The procedure is based on Eq. (12). The loading rate needs to be formulated as a function of the velocity whereafter a fitting algorithm can be applied. For this, we suggest the following approach:

1. Measure $F^{*}$ for different velocities $v$. It is possible to use only two different velocities but highly recommended that at least four different velocities are used to obtain better accuracy.

2. Find a relation between $F^{*}$ and $v$. Note that $r$ can be expressed as function of $v$ in an experiment, i.e.,

(a) Relate the loading rate $r$ to the velocity $v$ using Eq. 16.

(b) Use Eq. (12) to define a, possible implicit, relation between $F^{*}$ and $v$.

Using a and $\mathrm{b}$, there is a relation between $v$ and $F^{*}$ that depends only on $x_{\mathrm{b}}$ and $k_{\mathrm{off}}^{\mathrm{th}}$.

3. The parameter values $x_{\mathrm{b}}$ and $k_{\mathrm{off}}^{\text {th }}$ can now be assessed using a standard fitting procedure with the coupled values of $v$ and $F^{*}$.

\section{Numeric example using a WLC}

As a well-controlled example, we numerically simulated a force spectroscopy experiment of a receptor expressed on a tip of a polymer with WLC properties that was bound to an immobilized ligand. This simulation thus mimicked an experiment using AFM or OT instrumentation. The parameter values of the WLC model were set as; bond length, $x_{\mathrm{b}}$, $0.70 \mathrm{~nm}$, thermal off-rate, $k_{\mathrm{off}}^{\mathrm{th}}, 1.00 \times 10^{-4} \mathrm{~Hz}$, persistence length, $l_{\mathrm{p}}, 3.00 \mathrm{~nm}$, contour length, $L_{\mathrm{c}}, 10.0 \mu \mathrm{m}$, and thermal energy, $k_{\mathrm{b}} T, 4.11 \mathrm{pNnm}$. Since the elastic stiffness of the force probe, i.e., the AFM cantilever or the bead in the optical trap, is significantly higher than the elastic properties of the modeled WLC polymer, we modeled these as infinitely stiff. We thereafter analyzed all data closely following the approach described above:

1. The peak force $F^{*}$ was identified for four different velocities $v$. 

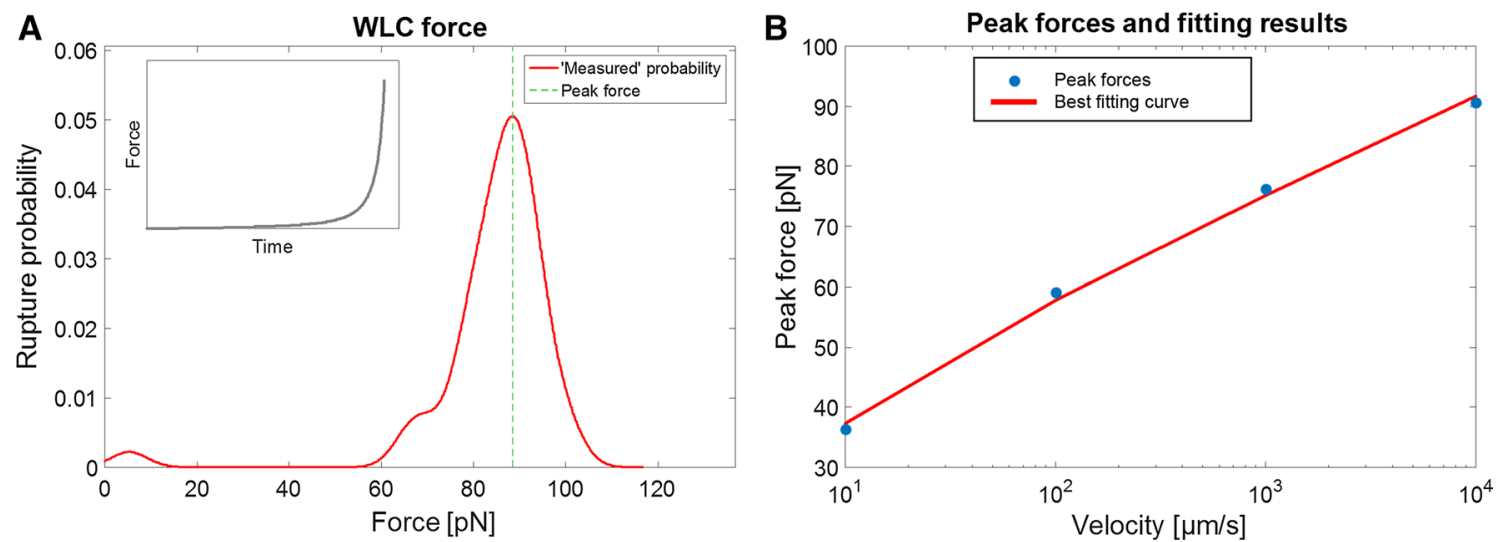

Fig. 2 a Spectrum of rupture forces obtained for $v=10,000 \mu \mathrm{m} / \mathrm{s}$ from 50 measurements. The peak force $88.47 \mathrm{pN}$. b Best estimates of the bond length and thermal off-rate obtained from a fitting algorithm based on data from four velocities and the corresponding peak forces

Table 1 The numerical results for the simulated example with the resulting values for the thermal off-rate and the bond length

\begin{tabular}{lllllll}
\hline$N$ & $v=10 \mu \mathrm{m} / \mathrm{s}$ & $v=100 \mu \mathrm{m} / \mathrm{s}$ & $v=1000 \mu \mathrm{m} / \mathrm{s}$ & $v=10,000 \mu \mathrm{m} / \mathrm{s}$ & $x_{\mathrm{b}}$ & $k_{\mathrm{off}}^{\text {th }}$ \\
\hline 50 & $39.57 \mathrm{pN}$ & $55.33 \mathrm{pN}$ & $74.58 \mathrm{pN}$ & $88.47 \mathrm{pN}$ & $0.685 \mathrm{~nm}$ & $1.54 \times 10^{-4} \mathrm{~Hz}$ \\
\hline
\end{tabular}

2. We calculated $t$, to be used in Eq. (15), for every $F^{*}$ with the corresponding velocity $v$ by using Eqs. (13) and (14). This relation between time and the force can also be readily measured during the experiments. With the time corresponding to the peak force and the loading rate function given by Eq. (15) we had everything we needed to use Eq. (12) as a relation between $F^{*}$ and $v$. This means that $F^{*}$ was expressed as a function of the velocity.

3. The acquired pair values for $F^{*}$ and $v$ were now used. A standard algorithm that finds the parameter values of the bond length and the thermal off-rate that minimizes the mean square error of the theoretical and measured values of $F^{*}$ was utilized.

For each of the four different extension velocities: 10, 100, 1000, and $10,000 \mu \mathrm{m} / \mathrm{s} ; 50 \mathrm{MC}$ force spectroscopy simulations were performed. The rupture forces were saved and four continuous rupture probability density distributions, using a Gaussian kernel density estimator $(\sigma=3 \mathrm{pN})$, were generated. The peak force was identified for each of the four distributions. Figure $2 \mathrm{a}$ shows the rupture force spectrum for the highest velocity. To estimate the bond length and thermal off-rate, we thereafter numerically fitted Eq. (12) to the data using a Nelder-Mead simplex algorithm to find the parameter values that minimized the mean square error of the peak forces. The data from the simulation are shown with the fitted values in Fig. $2 \mathrm{~b}$ and Table 1.

The assessed parameter values for this simulation are close estimates of the true values, where the bond length is underestimated with only $\sim 2.1 \%$, whereas the thermal off-rate is overestimated by $\sim 54 \%$. The discrepancy is expected due to the stochastic nature of the receptorbond complex and should therefore depend on the sample size. Since we conducted the analysis based on only 50 simulations, we expect this error to decrease significantly with increased sample size. To quantify the error in the parameters, we conducted a statistical analysis of the data (described below).

\section{Error dependencies of the sample size}

In the example above, measurement sets consisting of 50 rupture forces were used to identify the peak forces for each velocity. Due to the stochastic nature of the experiment, the accuracy is expected to be improved with larger data sets, i.e., the more rupture forces that are sampled, the less error there will be in the parameter values. To acquire acceptable accuracy in the parameter values, it is in generally recommended in the literature to conduct at least $\sim 50$ 100 rupture measurements (Evans 1999; Merkel et al. 1999; Björnham and Schedin 2009). Therefore, we performed simulations with 50, 70, 100, and 300 rupture forces, which allowed us to quantify the expected error in the parameters as a function of the sample sizes. In addition, a control set with ten million rupture forces for each velocity was performed. The resulting mean errors from these simulations for the bond length and the thermal off-rate are presented in Fig. 3 and Table 2. Since experiments normally are conducted with 50-300 samples, the mean relative error of the 


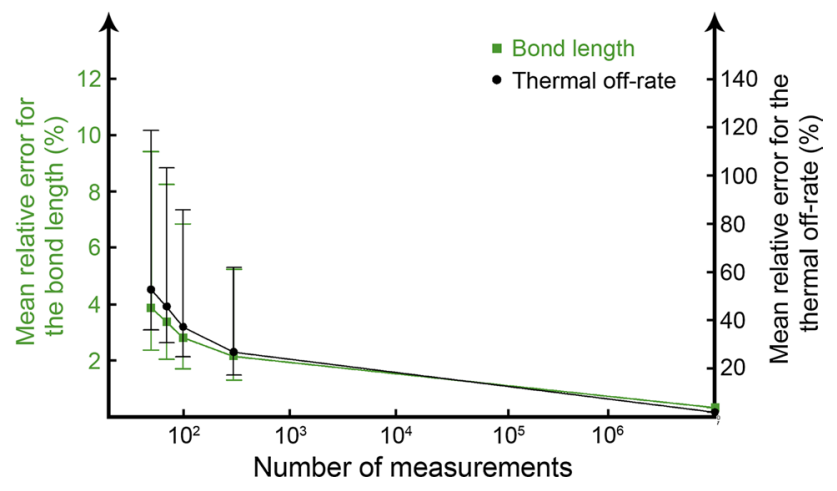

Fig. 3 Mean relative error of the parameters as function of number of measurements. The error bars show the quartiles of the stochastic distribution of retrieved parameter values

bond length is found to be less than $4 \%$ while the mean relative error in the thermal off-rate is $\sim 50 \%$. This difference in errors can be explained by the fact that the rupture force is significantly more sensitive to the bond length in comparison to the thermal off-rate, therefore this difference is less remarkable.

\section{Conclusions}

We have presented an extension of the standard DFS theory that can also accommodate nonlinear forces, denoted NLDFS. The NLDFS theory enables investigation of a wide range of biomechanical systems that show nonlinear force responses without compromising the well-established and frequently used linear DFS. Examples of receptorligand systems that can be analyzed using this theory are adhesins expressed on bacterial adhesion pili.

The data analysis using NLDFS requires a slightly more advanced fitting procedure than the conventional DFS theory to acquire the parameter values of the bond length and the thermal off-rate. The reason for this are that the loading rate becomes dynamic and the introduction of a new term, $(\mathrm{d} \ln r / \mathrm{d} t)$ in Eq. (12). This extra term, however, disappears for constant loading rates, which shows that the NLDFS theory reduces to the regular linear DFS for the case with a constant loading rate. In DFS, a set of different loading rates with the corresponding values of the peak forces are used.

To conduct the equivalent procedure in NLDFS, the protocol has to be modified. Instead of keeping the loading rate constant during experiments, the pulling velocity is kept constant. If the force increases linearly with distance, the loading rate in Eq. (16) is constant, and the NLDFS analysis falls into the linear DFS regime. This implies that the velocity can be used as the entity kept constant in measurements using both DFS and NLDFS theory. Finally, just as assumed in DFS experiment, we neglect the dynamic effects of the viscous drag force on the probe since the pulling velocities are slow.

Evans et al. refined the concept of DFS by introducing soft polymers linking the receptor-ligand bond (Evans and Ritchie 1999). By defining a compliance function, they compared how the peak force changed with and without a soft linker. Their approach allows for analysis of bond strengths in the presence of nonlinear external forces by defining the polymer force response using a relation between the probe stiffness and the characteristic stiffness of the polymers, and utilizing an apparent loading rate, which equals a constant probe stiffness multiplied by the pulling velocity. A theoretical correlation can thereafter be established, which is used in a curve-fitting procedure. The main concepts of that method and NLDFS presented in this work are similar. However, the NLDFS theory utilizes a more direct approach and introduces only a minimal modification of the linear DFS. Explicit information on the
Table 2 Statistical measures of the relative errors in the resulting parameter values in comparison to the analytic values

\begin{tabular}{|c|c|c|c|c|c|c|c|c|}
\hline \multirow[t]{2}{*}{ Method } & \multirow[t]{2}{*}{ Samples $N$} & \multirow[t]{2}{*}{ Iterations } & \multicolumn{4}{|c|}{ Mean relative error for the peak force $(\mu \mathrm{m} / \mathrm{s})$} & \multicolumn{2}{|c|}{$\begin{array}{l}\text { Mean relative } \\
\text { error }\end{array}$} \\
\hline & & & $v=10^{1}$ & $v=10^{2}$ & $v=10^{3}$ & $v=10^{4}$ & $x_{\mathrm{b}}$ & $k_{\mathrm{off}}^{\mathrm{th}}$ \\
\hline Monte Carlo & 50 & 10,000 & $5.42 \%$ & $3.13 \%$ & $2.26 \%$ & $1.81 \%$ & $3.88 \%$ & $52.7 \%$ \\
\hline Monte Carlo & 70 & 10,000 & $4.79 \%$ & $2.77 \%$ & $1.99 \%$ & $1.59 \%$ & $3.37 \%$ & $45.5 \%$ \\
\hline Monte Carlo & 100 & 10,000 & $4.17 \%$ & $2.38 \%$ & $1.76 \%$ & $1.41 \%$ & $2.81 \%$ & $37.3 \%$ \\
\hline Monte Carlo & 300 & 10,000 & $3.06 \%$ & $1.73 \%$ & $1.28 \%$ & $1.01 \%$ & $2.15 \%$ & $26.7 \%$ \\
\hline Monte Carlo & $10^{7}$ & 1 & $0.1 \%$ & $0.1 \%$ & $0.3 \%$ & $0.1 \%$ & $0.32 \%$ & $1.89 \%$ \\
\hline \multicolumn{3}{|c|}{$\begin{array}{l}\text { Analytical, most probable rupture forces } \\
(\mathrm{pN})\end{array}$} & 41.55 & 58.61 & 74.49 & 89.81 & - & - \\
\hline
\end{tabular}

The data were obtained by calculating the most probable rupture forces from $N$ measurements at four different velocities and the bond length and thermal off-rate were calculated using the method described in the theory section. This procedure was then repeated 10,000 times to quantify the expected errors in the parameter values 
stiffness of the probe and the soft linker in the system can be readily bypassed by direct investigation of the force vs. distance curve, which also implies that nonlinear responses in the probe are treated equally as nonlinear responses in the bio-complex linker. In other words, in the approach presented here, only the force experienced by the bond under investigation is considered, disregarding the origin of the force response, since it has no impact in the analysis.

Another approach to deal with nonlinear loading rates has been proposed elsewhere (Friedsam et al. 2003). Instead of using the peak force values, they used the probability density function for bond rupture. This expression is, however, rather complicated and depends on; the force, the loading rate, the bond length and the thermal off-rate, where the force and the loading rate distributions are found by investigating the experimental data. Estimates of the bond length and thermal off-rate may thereby be found by fitting the function to the rupture probability data. This method uses all data points, and not only the ones close to the peak force, which is of advantage since it makes use of a larger data set. On the other hand, the method is sensitive to outliers and measurement artifacts.

Besides the theoretical framework presented here, a protocol for how to conduct a practical measurement and data evaluation is described by a numerical example using Monte Carlo simulations. Since the rupture forces are stochastic, the parameter values will inherently have uncertainties coupled to them. We quantified the expected uncertainties by a large number of iterative simulations that provided the magnitude of errors that one would expect in a real experiment. It was found that already at 300 experimental data points the mean relative error for the bond length is only $\sim 2 \%$. However, in a real experiment using AFM or OT, additional measurement error and noise will be added on top of the inherent stochastic nature of the bond under investigation. Therefore, the values of the expected errors presented here are to be interpreted as a best-case outcome.

Acknowledgments This work was supported by the Swedish Research Council (2013-5379) and from the Kempe Foundation to M.A.

Open Access This article is distributed under the terms of the Creative Commons Attribution 4.0 International License (http://creativecommons.org/licenses/by/4.0/), which permits unrestricted use, distribution, and reproduction in any medium, provided you give appropriate credit to the original author(s) and the source, provide a link to the Creative Commons license, and indicate if changes were made.

\section{References}

Andersson M, Uhlin BE, Fällman E (2007) The biomechanical properties of $E$. coli pili for urinary tract attachment reflect the host environment. Biophys J 93:3008-3014. doi:10.1529/biophysj.107.110643
Bell G (1978) Models for the specific adhesion of cells to cells. Science 200:618-627. doi:10.1126/science.347575

Bianco P, Nagy A, Kengyel A et al (2007) Interaction forces between F-actin and titin PEVK domain measured with optical tweezers. Biophys J 93:2102-2109. doi:10.1529/biophysj.107.106153

Björnham O, Schedin S (2009) Methods and estimations of uncertainties in single-molecule dynamic force spectroscopy. Eur Biophys J 38:911-922. doi:10.1007/s00249-009-0471-8

Björnham O, Axner O, Andersson M (2008) Modeling of the elongation and retraction of Escherichia coli $\mathrm{P}$ pili under strain by Monte Carlo simulations. Eur Biophys J 37:381-391. doi:10.1007/s00249-007-0223-6

Björnham O, Nilsson H, Andersson M, Schedin S (2009) Physical properties of the specific PapG-galabiose binding in E. coli $\mathrm{P}$ pili-mediated adhesion. Eur Biophys J 38:245-254. doi:10.1007/ s00249-008-0376-y

Castelain M, Koutris E, Andersson M et al (2009) Characterization of the biomechanical properties of T4 pili expressed by Streptococcus pneumoniae - a comparison between helix-like and open coil-like pili. Chemphyschem 10:1533-1540. doi:10.1002/ cphc. 200900195

Castelain M, Ehlers S, Klinth JE et al (2011) Fast uncoiling kinetics of F1C pili expressed by uropathogenic Escherichia coli are revealed on a single pilus level using force-measuring optical tweezers. Eur Biophys J 40:305-316. doi:10.1007/s00249-010-0648-1

Chen F-J, Chan C-H, Huang Y-J et al (2011) Structural and mechanical properties of Klebsiella pneumoniae type 3 fimbriae. J Bacteriol 193:1718-1725. doi:10.1128/JB.01395-10

Evans E (1999) Looking inside molecular bonds at biological interfaces with dynamic force spectroscopy. Biophys Chem 82:8397. doi:10.1016/S0301-4622(99)00108-8

Evans E (2001) Probing the relation between force-lifetime-and chemistry in single molecular bonds. Annu Rev Biophys Biomol Struct 30:105-128

Evans E, Ritchie K (1997) Dynamic strength of molecular adhesion bonds. Biophys J 72:1541-1555. doi:10.1016/ S0006-3495(97)78802-7

Evans E, Ritchie K (1999) Strength of a weak bond connecting flexible polymer chains. Biophys J 76:2439-2447. doi:10.1016/ S0006-3495(99)77399-6

Forero M, Yakovenko O, Sokurenko EV et al (2006) Uncoiling mechanics of Escherichia coli type I fimbriae are optimized for catch bonds. PLoS Biol 4:1509-1516. doi:10.1371/journal. pbio.0040298

Friedsam C, Wehle AK, Khner F, Gaub HE (2003) Dynamic singlemolecule force spectroscopy: bond rupture analysis with variable spacer length. J Phys: Condens Matter 15:S1709-S1723. doi:10.1088/0953-8984/15/18/305

Kiss B, Karsai Á, Kellermayer MSZ (2006) Nanomechanical properties of desmin intermediate filaments. J Struct Biol 155:327-339. doi:10.1016/j.jsb.2006.03.020

Kramers HA (1940) Brownian motion in a field of force and the diffusion model of chemical reactions. Physica 7:284-304. doi:10.1016/S0031-8914(40)90098-2

Lee GU, Kidwell DA, Colton RJ (1994) Sensing discrete streptavidin biotin interactions with atomic-force microscopy. Langmuir 10:354-357. doi:10.1021/la00014a003

Merkel R, Nassoy P, Leung A et al (1999) Energy landscapes of receptor-ligand bonds explored with dynamic force spectroscopy. Nature 397:50-53. doi:10.1038/16219

Miller E, Garcia T, Hultgren SJ, Oberhauser AF (2006) The mechanical properties of $E$. coli type 1 pili measured by atomic force microscopy techniques. Biophys J 91:3848-3856. doi:10.1529/ biophysj.106.088989

Mortezaei N, Epler CR, Shao PP et al (2015a) Structure and function of enterotoxigenic Escherichia coli fimbriae from differing 
assembly pathways. Mol Microbiol 95:116-126. doi:10.1111/ mmi. 12847

Mortezaei N, Singh B, Zakrisson J et al (2015b) Biomechanical and structural features of CS2 fimbriae of enterotoxigenic Escherichia coli. Biophys J 109:49-56. doi:10.1016/j.bpj.2015.05.022

Neuert G, Albrecht C, Pamir E, Gaub HE (2006) Dynamic force spectroscopy of the digoxigenin-antibody complex. FEBS Lett 580:505-509. doi:10.1016/j.febslet.2005.12.052

Strick TR, Dessinges M-N, Charvin G et al (2002) Stretching of macromolecules and proteins. Rep Prog Phys 66:1-45. doi:10.1088/0034-4885/66/1/201
Strunz T, Oroszlan K, Schäfer R, Güntherodt HJ (1999) Dynamic force spectroscopy of single DNA molecules. Proc Natl Acad Sci USA 96:11277-11282. doi:10.1073/pnas.96.20.11277

Sulchek TA, Friddle RW, Langry K et al (2005) Dynamic force spectroscopy of parallel individual Mucin1-antibody bonds. Proc Natl Acad Sci USA 102:16638-16643. doi:10.1073/ pnas.0505208102

Yuan C, Chen A, Kolb P, Moy VT (2000) Energy landscape of streptavidin-biotin complexes measured by atomic force microscopy. Biochemistry 39:10219-10223. doi:10.1021/bi992715o 